\title{
The Grand Challenges in Cardiovascular Drug Delivery
}

\author{
Iwona Cicha* \\ Cardiovascular Nanomedicine Unit, Section of Experimental Oncology und Nanomedicine (SEON), Else Kröner-Fresenius- \\ Stiftung-Professorship, ENT-Department, Universitätsklinikum Erlangen, Erlangen, Germany
}

Keywords: atherosclerosis, thrombosis, new drugs, nanocarriers, personalization, translation

Despite the ongoing development in invasive cardiovascular interventions and pharmacological therapies over the past 25 years, cardiovascular diseases (CVD) continue to account for $31 \%$ of all deaths worldwide. This amounts to 17.9 million deaths per year, a number that is expected to grow to more than 23.6 million by 2030, according to WHO estimates. According to the European Cardiovascular Disease Statistics 2017 (Wilkins et al., 2017), CVD caused 3.9 million deaths in Europe alone. As reported by the 2019 Heart Disease and Stroke Statistics of the American Heart Association (Benjamin et al., 2019), every $40 \mathrm{~s}$, an American will suffer from a myocardial infarction (MI).

In the recent years, effective medicines allowed to better control blood cholesterol levels, to lower blood pressure and, finally, to reduce inflammation, which contributed to improved management of patients with CVD. However, the first symptom of CVD in more than $50 \%$ cases is acute cardiovascular event or sudden cardiac death. This background calls for more reliable risk assessment allowing earlier disease detection and for improved therapeutic approaches.

Atherosclerosis and arterial thrombosis, the underlying causes of cardiovascular morality, can manifest as acute coronary syndromes, stroke or peripheral arterial disease. Drug delivery systems, which enable for example targeted application of medicines should have an enormous impact for the affected patients, as personalized tools to support the physicians in both the choice and the administration of recommended therapies. Among the potential therapeutic targets of novel

OPEN ACCESS

Edited and reviewed by:

David J. Brayden,

University College Dublin, Ireland

*Correspondence: Iwona Cicha

Iwona.Cicha@uk-erlangen.de

Specialty section:

This article was submitted to Cardiovascular Drug Delivery,

a section of the journal

Frontiers in Drug Delivery

Received: 28 September 2021 Accepted: 11 October 2021 Published: 25 October 2021

Citation:

Cicha I (2021) The Grand Challenges in Cardiovascular Drug Delivery. Front. Drug. Deliv. 1:784731. doi: 10.3389/fddev.2021.784731 drug delivery systems and biomaterial implants are vulnerable atherosclerotic plaques, ischemic myocardium, cerebro-, retino-, and renovascular disorders, but also thrombosis. Besides, cardiovascular stents, prostheses and patches can serve as tools for local drug delivery to enhance vascular healing and prevent neoatherosclerosis. The road from the ambitious experimental ideas to the clinical routine remains difficult, however. There are several Grand Challenges that must be addressed to overcome the hurdles for the development of drug delivery systems that will improve the outcomes of patients with CVD. Besides the biggest obstacle, which is the clinical safety and translation, also innovation, personalization and drug delivery on-demand represent major challenges. These challenges are discussed in detail below.

\section{INNOVATION}

Understanding the basic molecular mechanisms underlying the development and destabilization of atherosclerotic plaques helped to establish therapeutic standards for patients with CVD. Starting with e.g. hydroxy-methyl-glutaryl $\mathrm{CoA}$ (HMG-CoA) reductase, the key enzyme that has been exploited as a pharmacological target of statins, the combination of basic and clinical research contributed to the development of novel anti-atheroslerotic drugs, including protein convertase subtilisin/kexin type 9 (PCSK9) inhibitors. Recent trials with anti-inflammatory antibodies (i.e. CANTOS trial using canakinumab/anti-IL $1 \beta$ ) were based on understanding of the inflammatory background of atherosclerosis and have shown positive outcomes in affected patients. Innovative 
approaches are also required to improve the therapies of $\mathrm{MI}$ and heart failure, resulting from the rupture of coronary atherosclerotic plaques. Despite the advancement in patient care, ischemic heart disease is still the leading cause of death worldwide. The existing pharmacological interventions aim to preserve and improve ventricular function, but are primarily symptomatic and unable to restore the myocardial tissue once the permanent damage to the left ventricle occurred. Novel small molecule drugs, biologics and regenerative strategies addressing ischemic myocardium are therefore urgently needed.

Thus, deepening the knowledge of the disease, in parallel with continuing medicinal chemistry and pharmacology research, should contribute to the discovery of novel drugs to suppress atherosclerotic, atherothrombotic and ischemic processes. Pharmacologic substances alone, however, may suffer from poor bioavailability, short half-lives in vivo, being prone to protease damage, insufficient stability upon storage, or high production costs. Innovation is thus also needed with respect to the development of novel carriers, including nanocarriers, polymers, or hydrogels for defined local delivery, which have been in the focus of some EU-funded projects in the past [NanoAthero Project (Chauvierre and Letourneur, 2015; Cicha et al., 2018)] and now (Cupido Project, https://www. cupidoproject.eu/). Innovative devices and approaches to drug delivery should greatly improve the efficacy and clinical utility of the novel therapies.

\section{PERSONALIZATION}

One of the Grand Challenges in medicine in general, and cardiovascular drug delivery in particular, is the need of personalized therapies. Currently, medicine adapts to the needs of the individual patients in a lengthy try-and-err process, whereby the standard therapeutic regimen showing effects in large cohorts is applied and subsequently adapted, depending on patient's responses and the outcome. At the same time, it is clear, that every patient comes with their individual disease burden, risk factors, genetic background and life style environment.

In order to improve the clinical decision-making and therapeutic regimen, it would therefore be necessary to employ extensive diagnostics including proteomics, DNA sequencing, and imaging, ideally in combination with artificial intelligencesupported data mining and wireless health monitoring devices, in order to characterize the vulnerability status of the patient and gain information about the disease burden and localization. This process should allow moving from systemic medication towards the targeted therapy tailored to the patient's needs. One of the examples illustrating such need in the cardiovascular field is the pharmacological therapy of ischemic stroke, using an enzyme tissue plasminogen activator (tPA), the only thrombolytic drug approved for this indication by FDA since 1996 (Chapman et al., 2014). The recommended thrombolytic therapy in patients with acute ischemic stroke, involves systemic administration of a fixed dose of tPA $(0.9 \mathrm{mg} / \mathrm{kg}$, maximum $90 \mathrm{mg})$ within $4.5 \mathrm{~h}$ of stroke onset (rt-PA Stroke Study Group, 1995). The use of tPA is strongly limited both by the narrow eligibility and administration window, and by the risk of hemorrhagic complications (Miller et al., 2011). Another drawback is the use of fixed dose for intravenous infusion of tPA, which may result in a situation, where the administered dose is too low in some patients, resulting in insufficient efficacy. At the same time, the fixed dose may be too high for other patients, causing lifethreatening bleeding complications. Thus, strategies are urgently needed that allow adaptation of the drug dose to the individual needs of the patient and/or targeted applications of e.g. plasminogen activators. Personalized drug delivery is expected in a longer term to minimise side effects and improve treatment efficacy in patients with CVD.

\section{DELIVERY ON DEMAND}

The key drawbacks of pharmacologic therapies are their considerable side effects and low efficacy at the tolerated dose. These drawbacks result from systemic administration of small molecule drugs or biologics, so that their bioavailability or concentration in the diseased regions may be too low to induce a meaningful improvement. At the same time, the systemic administration means that the medicines circulate in the bloodstream, affecting the metabolism and function of healthy tissues, which can result in off-target adverse effects. Controlled, "on-demand" drug delivery has the advantage of disease-specific treatment and allows reducing off-target effects by a stimuli-mediated drug release in the affected region. This is particularly important in case of atherosclerosis and thrombosis, as well as their clinical manifestations including myocardial infarction and stroke, which represent localized pathological processes. Although insufficient response to systemicallyadministered drugs is a common problem in CVD patients, producing systems for drug delivery on demand is far from trivial.

The process of drug encapsulation in nanoparticles or biding them to carriers can strongly increase drug bioavailability and circulation time, resulting (albeit unspecifically) in improved drug concentrations at the affected sites. However, the use of (nano)carriers does not guarantee disease-specific drug accumulation, or the proper control of the drug release. In order to achieve this, much more complex delivery systems are required, which incorporate molecules reacting to specific stimuli, as well as targeting agents or ligands. Upon accumulation in the target tissue, such "smart" drug carriers release they payload in response to specific stimuli, which may be intrinsic (e.g. disease-related, usually involving chemical and biological factors, such as ionic strength, $\mathrm{pH}$, reactive oxygen species, enzymatic reactions, or biomechanical forces), or externallyapplied (such as light, ultrasound, magnetic or electrical fields directed at a specific region of the body). Such "smart" responsive drug carriers, utilizing stimuli specific to the microenvironment of atherosclerotic plaque, ischemic myocardium or occluded cerebral vessel, would be especially suitable for cardiovascular applications and could improve treatment efficacy, minimize toxicity and enhance sensitivity towards pathologic processes. However, although the development of such complex carriers is 
already possible with existing technologies, there are multiple challenges related to their potential clinical use (Cicha et al., 2018). In particular the costs of GMP-compliant production and upscaling, the necessity of selecting safe and biocompatible components, as well as the extensive characterization and testing to meet the safety requirements necessary for regulatory approval represent major obstacles for stimuliresponsive drug delivery systems. Some of these safety and translation hurdles are discussed in detail below.

\section{CLINICAL SAFETY AND TRANSLATION}

Targeted delivery of small molecule drugs, biologics (cell-, growth factor- or RNA-based therapeutics) and advanced therapeutic modalities ("smart" drug delivery (nano)systems) represents a novel approach to CVD, with high potential for reducing the burden of atherosclerosis, improving the ventricular function in patients with myocardial infarction and heart failure, and improving outcomes in patients with ischemic stroke. Unfortunately, despite the highly promising preclinical data from animal studies, their clinical translation is hampered by challenges related to the complexity of drug development and manufacturing, safety/tolerability, as well as regulatory pathway requirements. It must be noted that these challenges are faced also by the cardiovascular drugs, which do not contain an advanced delivery system. Among new promising drugs that failed to reach the clinic in the last 5 years was a recombinant apolipoprotein A-I Milano (MDCO-216) discontinued after phase II trials, as well as bococizumab, a humanized monoclonal antibody against PCSK9 developed by Pfizer and losmapimod, an inhibitor of p38 mitogen-activated protein kinases, developed by GSK and discontinued after phase III trials (Li et al., 2019). Although the reasons for discontinuation are diverse, it is clear that the later the phase of development, the higher are the costs of candidate drug's failure. Generally, in the process of development of drug delivery systems, drug-target interaction modelling, extensive in vitro toxicity studies and good predictive animal models are of critical importance to optimize the properties of the candidates. The challenges related to scale-up of experimental drug delivery systems, their GMP-compliant manufacturing, quality control, documentation and packaging, should not be underestimated. Further, immuno-safety of drug delivery systems, as well as cardiovascular risks related to their intravascular administration must be evaluated in detail to exclude the risk immunogenic side effects (Cicha et al., 2018). One of the major translation hurdles is also completion of clinical trials within a reasonable time, as recruiting a large number of patients and the subsequent observation period may take several years. All these requirements may present a hurdle that is far too

\section{REFERENCES}

Benjamin, E. J., Muntner, P., Alonso, A., Bittencourt, M. S., Callaway, C. W., Carson, A. P., et al. (2019). Heart Disease and Stroke Statistics-2019 Update: A high for e.g. academia spin-off companies, resulting in a poor success of cardiovascular drug delivery systems in terms of approval. The scale of difficulties is also reflected in a small number of ongoing clinical trials that involve nanoparticle-based drug delivery to atherosclerosis. Currently, only 2 ongoing phase II trials are registered that address drug delivery to atherosclerosis, one involving paclitaxel encapsulated in LDLlike nanoparticles (PAC-MAN trial) for the treatment of patients with coronary and aortic atherosclerosis and the other utilizing cholesterol-rich lipid nanoparticles loaded with an antiinflammatory agent methotrexate in patients with stable coronary disease (https://clinicaltrials.gov/). As the development of effective drug delivery systems is very complex, time consuming and bears a considerable financial risk, new partnerships between academia, biotech industry and big pharmaceutical companies will be necessary to bring novel therapeutic modalities to the market. New in silico methods and predictive toxicology models should in the future assist the scientist in making new discoveries and translating them into the cardiovascular drug delivery systems.

\section{CONCLUSION}

In the light of the growing numbers of patients suffering from CVD, novel drug delivery systems and targeting strategies for therapeutic agents are urgently needed. Innovative experimental approaches have been previously reported, including e.g. targeted delivery of small molecule drugs, biologics, RNA-based therapeutics, or stem cells, which showed promising preclinical results. These studies utilized delivery of therapeutics loaded into biocompatible nanoparticles, as well as biomaterial-based carriers for local drug release, representing an important step towards the development of disease-targeted, personalized therapies. However, until now, the benefits of new scientific discoveries and advanced technological approaches have not been realized in clinical practice. At the same time, it becomes clear that more efforts, commitment and investment are necessary to speed up the testing, translation and commercialization of these novel and potent cardiovascular therapies. Continuous efforts to address the challenges in the field, which are also in the focus of the Specialty Section on Cardiovascular Drug Delivery, should help in the translation of new therapeutics and decrease the global burden of cardiovascular diseases.

\section{AUTHOR CONTRIBUTIONS}

The author confirms being the sole contributor of this work and has approved it for publication.

Report from the American Heart Association. Circulation 139 (10), e56-e528. doi:10.1161/CIR.0000000000000659

Chapman, S. N., Mehndiratta, P., Johansen, M. C., Mcmurry, T. L., Johnston, K. C., and Southerland, A. M. (2014). Current Perspectives on the Use of Intravenous Recombinant Tissue Plasminogen Activator (tPA) for Treatment of Acute 
Ischemic Stroke. Vasc. Health Risk Manag. 10, 75-87. doi:10.2147/ VHRM.S39213

Chauvierre, C., and Letourneur, D. (2015). The European Project NanoAthero to Fight Cardiovascular Diseases Using Nanotechnologies. Nanomedicine 10 (22), 3391-3400. doi:10.2217/nnm.15.170

Cicha, I., Chauvierre, C., Texier, I., Cabella, C., Metselaar, J. M., Szebeni, J., et al. (2018). From Design to the Clinic: Practical Guidelines for Translating Cardiovascular Nanomedicine. Cardiovasc. Res. 114 (13), 1714-1727. doi:10.1093/cvr/cvy219

Li, T., Jiang, S., Ni, B., Cui, Q., Liu, Q., and Zhao, H. (2019). Discontinued Drugs for the Treatment of Cardiovascular Disease from 2016 to 2018. Int. J. Mol. Sci. 20 (18). doi:10.3390/ijms20184513

Miller, D. J., Simpson, J. R., and Silver, B. (2011). Safety of Thrombolysis in Acute Ischemic Stroke: a Review of Complications, Risk Factors, and Newer Technologies. The Neurohospitalist 1 (3), 138-147. doi:10.1177/1941875211408731

rt-PA Stroke Study Group, The National Institute of Neurological Disorders and Stroke (1995). Tissue Plasminogen Activator for Acute Ischemic Stroke. N. Engl. J. Med. 333(24), 1581-1587. doi:10.1056/ NEJM199512143332401
Wilkins, E., Wilson, L., Wickramasinghe, K., et al. (2017). European Cardiovascular Disease Statistics 2017.

Conflict of Interest: The author declares that the research was conducted in the absence of any commercial or financial relationships that could be construed as a potential conflict of interest.

Publisher's Note: All claims expressed in this article are solely those of the authors and do not necessarily represent those of their affiliated organizations, or those of the publisher, the editors and the reviewers. Any product that may be evaluated in this article, or claim that may be made by its manufacturer, is not guaranteed or endorsed by the publisher.

Copyright (c) 2021 Cicha. This is an open-access article distributed under the terms of the Creative Commons Attribution License (CC BY). The use, distribution or reproduction in other forums is permitted, provided the original author(s) and the copyright owner(s) are credited and that the original publication in this journal is cited, in accordance with accepted academic practice. No use, distribution or reproduction is permitted which does not comply with these terms. 